\title{
The effects of combined training on bone metabolic markers in postmenopausal women
}

\section{Les effets d'un entraînement combiné sur les marqueurs du métabolisme osseux chez la femme post-ménopausée}

\author{
A. Pereira ${ }^{a, b, *}$, A.M. Costa ${ }^{b, c, d}$, A. Palmeira-de-Oliveira ${ }^{d, e, f}$,

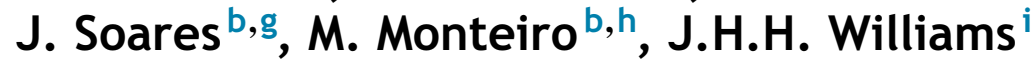 \\ a School of Education, Polytechnic Institute of Setubal, Setúbal, Portugal \\ ${ }^{\mathrm{b}}$ Research Center in Sports Sciences, Health Sciences and Human Development (CIDESD), Vila Real, Portugal \\ c Department of Sport Sciences, University of Beira Interior, Covilhã, Portugal \\ ${ }^{\mathrm{d}}$ Health Sciences Research Centre (CICS-UBI), Covilhã, Portugal \\ e Faculty of Health Science, University of Beira Interior, Covilhã, Portugal \\ ${ }^{\mathrm{f}}$ Health Products Research and Development (Labfit - HPRD), Covilhã, Portugal \\ g Department of Sport Sciences, Exercise and Health, University of Trás-os-Montes and Alto Douro, Vila \\ Real, Portugal \\ h School of Education, Polytechnic Institute of Bragança, Bragança, Portugal \\ i Chester Center for Stress Research, Institute of Medicine, University of Chester, UK
}

Received 29 April 2015; accepted 8 March 2016

Available online 4 May 2016

\section{KEYWORDS \\ Exercise; \\ Bone formation \\ marker; \\ Post-menopausal \\ women}

\begin{abstract}
Summary
Objectives. - Exercise plays an important role in maximizing and subsequent reduction of the maximum rates of bone loss. The purpose of this study was to determine whether 16 weeks of combined exercise in postmenopausal would affect bone metabolism.

Equipment and methods. - Eleven participants (53.1 $[ \pm 4.0]$ years) performed combined training that consisted of $60-75 \%$ of 1 RM, $2-3$ sets of $10-15$ repetitions in specific machines and 20-30 min of cardiovascular exercises using an step platform (55-80\% FCreserve). Cross-linked $\mathrm{N}$-terminal telopeptides of type I collagen (NTX) was used to analyze bone resorption and serum alkaline phos-phatase (ALP) to analyze bone absorption.
\end{abstract}

\footnotetext{
* Corresponding author. School of education at the polytechnic, institute of Setúbal, 2910-504 Setúbal, Cidesd Vila Real, Portugal. E-mail address: ana.pereira@ese.ips.pt (A. Pereira).
} 
Results. - After the training period, postmenopausal significantly $(P<0.05)$ increase their muscle functionality (14\% to $26.5 \%$ ). Levels of NTX were not affected but there was a significant increase in bone synthesis (ALP: $12.5 \%$ to $15.9 \%$ ), while there is no change in bone resorption. Combined training appears to be useful in improving total muscular performance and potentially in bone density.

(c) 2016 Elsevier Masson SAS. All rights reserved.

\section{MOTS CLÉS}

Exercice ;

Le marqueur de la

formation osseuse ;

Les femmes

post-ménopausées

\section{Résumé}

Objectifs. - L'exercice joue un rôle important dans l'optimisation et la réduction subséquente des taux maximales de la perte osseuse. Cette étude avait comme objectif déterminer si après 16 semaines d'exercice combiné, en postménopause, le métabolisme osseux serait affecté. Matériel et méthodes. - Onze participants $(53,1[ \pm 4,0]$ ans) ont réalisé une formation combinée composée de $60-75 \%$ de $1 \mathrm{RM}, 2-3$ séries de $10-15$, reproduite dans des machines spécifiques, et 20-30 min d'exercices cardiovasculaires utilisant un step fitness (55-80\% FCreserve). Un réticulé télopeptides $\mathrm{N}$-terminal du collagène de type I (NTX) a été utilisé pour analyser la réabsorption des os et sérum alcaline phos-phatase (ALP) pour analyser l'absorption de l'os.

Résultats. - Après la période de formation, les femmes post-ménopausées ont vu la fonctionnalité des muscles augmenter significativement $(p<0,05)(14-26,5 \%)$. Les niveaux de NTX n'ont pas été affectés, mais il y avait une augmentation significative de la synthèse osseuse (ALP : 12,5-15,9\%), alors qu'il n'y a aucun changement dans la réabsorption osseuse. La formation combinée semble être utile dans l'amélioration de la performance musculaire totale et, potentiellement, dans l'augmentation de la densité osseuse.

(c) 2016 Elsevier Masson SAS. Tous droits réservés.

\section{Introduction}

There is growing evidence that the decline in estrogen levels caused by menopause induces a state of negative metabolic changes in which bone resorption often exceeds bone formation, thereby decreasing bone mass [1,2]. During the postmenopausal period, typically 50-60 years of life, muscular strength also declines as bone metabolism changes result in reduced bone density $[3,4]$. Moreover, with aging and the resulting sedentary lifestyle, results in physiological changes responsible for losses in motor function. Thus, there is an increasing need for novel techniques to counteract bone and muscular loss.

Studies on exercise training suggest that it may play an influential role preventing at least some of the negative consequences of the menopause, such as osteoporosis [5]. A positive effect of exercise on bone mass has been demonstrated in postmenopausal women [6-9] although the results were not always consistent due to methodological issues, namely: the age of the participants, differences between each mode of exercise (aerobic vs. resistance exercise, isometric or concentric actions, frequency of exercise, testing measures), the duration of the intervention and the markers of bone changes used.

Bone density is determined by a balance between bone synthesis by osteoblasts and resorption by osteoclasts [10]. To evaluate the effect of distinct factors on bone metabolism, different bone markers have been identified [11]. The urinary cross-linked $\mathrm{N}$-terminal telopeptides of type I collagen (NTX) is considered a specific bone resorption marker [2] and bone-alkaline phosphatase (ALP), is a recognized bone formation marker [12]. While exercise seems to be effective in lowering the risk of falls and maintain muscular performance in selected people and it should through alterations in regulation of bone metabolism prevent the deterioration [6]. It is well accepted that high-intensity aerobics and high-speed resistance training exercise seems to be more efficient to increase bone metabolism than low- to moderate-intensity activity $[5,6]$. Thus, power training in postmenopausal women could be important for the maintenance of bone mineral density as an easily available effective exercise programs. Muscle strength has been suggested to increase bone integrity and the ability to withstand the biomechanical forces involved in muscular tasks [13]. However, muscle power is more closely associated to functional task performance and risk of falling in postmenopausal women than maximal strength [14]. The aim of this study was to examine the effects of 16 weeks of combined three-weekly step aerobics exercise and highspeed power training on maximal strength of the lower- and upper-body extremities. It was hypothesized that specific combined training in postmenopausal women would lead to significant gains in muscular performance and alter the balance in bone metabolism towards synthesis as opposed to resorption.

\section{Methods}

\subsection{Participants}

Eleven postmenopausal Caucasian women (age: $53.1 \pm 4.0$ years; body mass: $68.2 \pm 11.2 \mathrm{~kg}$; height: $1.55 \pm 0.06 \mathrm{~m}$ ) were submitted to a combined exercise 
program. Although isn't an older sample the study but is relevant to the understanding of the effects of exercise on bone metabolism. In addition, prevention should be a principle in practice and choose the type of exercise in the older population. Apart from routine daily tasks, the group underwent a 16-week resistance-training program of three training sessions per week in non-consecutive days. None of the participants had a history of resistance training. Before inclusion in the study, all candidates were thoroughly screened by a physician. None of the participants were past of smokers and had a history of hormone (estrogen) replacement therapy or had ever taken medication that affects bone metabolism, such as glucocorticoid, warfarin, methotrexate, bisphosphonate, vitamin $\mathrm{D}$, vitamin $\mathrm{K}$, or calcitonin. A written informed consent was obtained from each participant. The experimental procedures were approved by the university local research ethics committees, following the Helsinki declaration.

\subsection{Testing procedures}

The evaluation process requires reliability, specificity and facility of application, especially when participants are sedentary and inexperienced. Thus, we used protocols that were time-economical and that had been previously used in recent studies for the assessment of muscle-skeletal function in older people [15]. All testing procedures were applied before the experimental period (T1) and after 16 weeks of training (T2). Measurements (T1 and T2) took place over a period of 3 days (three sessions separated by 3 to 5 days), always in the same location and time and supervised by the same researchers. In the first session, all participants were assessed on anthropometric variables: weight, height, resting heart rate and functional parameters (sit-to-stand and timed and go up tests). The second session (3rdday) involved measures of power (vertical jump) and maximum dynamic strength (one-repetition maximum leg extension and bench press). Before testing, each subject was familiarized with all strength testing procedures, preceded by a general warm-up routine. Verbal encouragement was given throughout the voluntary test and biofeedback provided in order to maximize motivation.

\subsection{Anthropometric measures}

Total height $(\mathrm{cm})$ and body weight $(\mathrm{kg})$ were assessed according to international standards for anthropometric assessment. To evaluate height $(\mathrm{cm})$ a stadiometer (SECA, model 225, Germany) with a scale range of $0.10 \mathrm{~cm}$ was used and body mass $(\mathrm{kg})$ was measured to the nearest $0.1 \mathrm{~kg}$ using a digital scale (Philips, type HF 351/00). These parameters were assessed prior to any physical performance test. Participants were tested whilst wearing shorts and t-shirts (shoes and socks were removed).

\subsection{Strength tests}

Lower-body maximal strength was assessed using onerepetition maximum leg-extension $\left(1 \mathrm{RM}_{\mathrm{LE}}\right)$ [3]. On command, the subject performed a concentric leg extension (as fast as possible) starting from the flexed position to reach the full extension of $180^{\circ}$ against a resistance determined by given weight plates following 4 to 5 separate attempts until the subject was unable to extend the legs to the required position. The last acceptable extension with the highest possible load was determined as 1 RM $\mathrm{LE}_{\mathrm{LE}}$.

Additionally, each subject was tested for maximal bilateral concentric one-repetition bench press $\left(1 \mathrm{RM}_{\mathrm{BP}}\right)$. The subject was instructed to perform from the starting position a purely concentric action maintaining the shoulders in a $90^{\circ}$ abducted position following 3 to 4 trials until the subject was unable to reach the fully extended position of the arms. The last acceptable extension with the highest possible load was determined as $1 \mathrm{RM}_{\mathrm{BP}}$ [15].

\subsection{Power tests}

A trigonometric carpet (Ergojump Digitime 1000; Digitest, Jyvaskyla, Finland) was used to assess maximum height in counter-movement vertical jump (CMJ) [15]. Each subject started from an erect standing position and the end of the concentric phase corresponded to a full leg extension $\left(180^{\circ}\right)$. The test was performed three times, each one separated by a 2-minute of resting period. The average maximum height of three trials was adopted and expressed in centimeters $(\mathrm{cm})$.

\subsection{Functional tasks}

The 8-foot up and -go test (TUG) was used to assess the dynamic balance, represented by the total time required for the participants to rise from a seated position, walk eight feet $(2.44 \mathrm{~m})$, turn and return to a seated position [16].

Additionally, the 30-second sit-to-stand test (STS) was performed [16]. Before starting a straight-backed chair was placed next to a wall; participants were asked to flex their arms across the chest and to stand up from a seated position on the chair. From the sitting position, the subject stood up to full height, then completely back down, this cycle being repeated as quickly as possible for 30 seconds. The total number of repetitions was recorded [17].

\subsection{Bone marker measurements}

Two bone markers were tested: cross-linked N-telopeptide of type I collagen and the bone alkaline phosphatase [6]. The NTX-Serum test ELISA was used for the quantitative measured of cross-linked $\mathrm{N}$-telopeptide of type I collagen in human serum. It was performed using the commercial test Osteomark $^{\circledR}$ (Tecomedical AG, Switzerland) following the instructions supplied with the test. The bone alkaline phosphatase (also called skeletal alkaline phosphatase [sALP]) was performed using the Access Ostase assay, an in vitro one-step immunoenzymatic assay for the quantitative measurement of sALP, using Beckman Coulter Access Ostase Assay (Beckman Coulter Inc., Fullerton, CA). Assays were carried out following manufacturer's instructions. 


\subsection{Combined exercise program}

The training protocol included the step aerobics exercise that consisted of 3 sessions per week, 45 to 60 minutes per session during 16 weeks with the same certified instructor. The aerobic component within the training program included 30 minutes of cardiovascular exercises using a step platform. Each session included 10 minutes of warm-up and 20 minutes of step aerobics training. In the first 2 weeks of exercise, participants were initiated to the basic movements and choreography with continuous movement of legs and alternating movement of the arms (bicep curls and lateral raises at shoulder level and above the head) simultaneously with the selected steps. Exercise included movements of conventional basic step. The aerobic session started with a work heart rate $(\mathrm{HR})$ of $40-50 \%$ of maximum $\mathrm{HR}\left(\mathrm{HR}_{\max }\right)$ [18] (1-3th week), increasing progressively to $50-70 \% \mathrm{HR}_{\max }$ (4-6th week), and then to 70-85\% HR (7-8th week). The perceived exertion was 11 to 13 (6 to 20 points in Borg scale). HR monitoring equipment (Accurex plus, Polar Electro Oy, Finland) was used to monitor and records were kept during exercise. Music was selected to increase work HR and the cadence of the sessions was between 120 and 128 foot-strikes per minute. Then, participants performed 2 exercises for the leg extensor muscles (leg extension and leg press) and 1 exercise for the arm extensor muscles (the bench press) and finished with abdominal crunches and trunk extensors. An interval period of at least 2 min was permitted between sets and between exercises. During the first 2 weeks of the training period, the participants trained with loads of $40-50 \%$ of the individual $1 \mathrm{RM}, 10-12$ repetitions per set, and 3-4 sets of each exercise, and progressively increase to $75 \%$ of 1 RM, 3 sets by $4-6$ reps. Participants were performing all exercises at high velocity, with instructions to do them "as fast as you can". Each training session was closely supervised and monitored by two researchers, specialized in physiology and in aerobic-dance instruction to direct and assist each subject towards achieving the appropriate work rates and loads [3].

\subsection{Statistical analysis}

Data are presented as descriptive statistics by mean and standard deviation $(\mathrm{x} \pm \sigma)$ were calculated for all variables. Percent changes from before to after were calculated from the differences in the scores. Comparisons of means at baseline and after exercise training were performed using a one-sample $t$-test and paired simple $t$-test. A $P$-value, set a priori, of $P \leq 0.05$ was considered statistically significant. Data were analyzed using SPSS 21.0 for Windows.

\section{Results}

A significant $F$-value was observed $(F=46.0, P=0.000)$ between all measures. Test-retest reliabilities, as showed by ICC, ranged from 0.89 to 0.93 for all testing exercises. There were no significant differences observed for anthropometric, in height, weight or BMI between first (T1) and second evaluation (T2) in the postmenopausal women (Table 1). From the pre- to post-training period, the post-menopausal significantly $(t=-2.951 ; P=0.015)$
Table 1 Upper and lower limbs strength and functional capacity in postmenopausal women before and after 16 weeks of combined training.

\begin{tabular}{|c|c|c|c|}
\hline & $\mathrm{T} 1$ & $\mathrm{~T} 2$ & $P$ \\
\hline Measurement & $\mathrm{x} \pm \sigma$ & $\mathrm{x} \pm \sigma$ & $(\mathrm{T} 1-\mathrm{T} 2)$ \\
\hline $1 \mathrm{RM}_{\mathrm{BP}}(\mathrm{kg})$ & $21.8 \pm 7.5$ & $25.0 \pm 5.0$ & 0.240 \\
\hline $1 \mathrm{RM}_{\mathrm{LE}}(\mathrm{kg})$ & $52.2 \pm 5.6$ & $59.5 \pm 6.5$ & 0.015 \\
\hline CMJ (cm) & $12.8 \pm 2.1$ & $15.7 \pm 3.7$ & 0.047 \\
\hline TUG (sec.) & $22.9 \pm 8.2$ & $23.3 \pm 7.1$ & 0.001 \\
\hline STS (no.) & $23.8 \pm 4.4$ & $30.1 \pm 3.9$ & 0.014 \\
\hline
\end{tabular}

Data are presented as mean \pm standard deviation $(\mathrm{x} \pm \sigma)$; $P$ (T1-T2): $P<0.05$. Significantly different from before (T1) to after (T2) training; $1 \mathrm{RM}_{\mathrm{BP}}$ : one-repetition bench press; $1 \mathrm{RM}_{\mathrm{LE}}$ : one repetition maximum leg-extension; $\mathrm{CMJ}$ : countermovement vertical jump; TUG: 8-foot-up and -go test and STS: 30 -second sit-to-stand test.

increased their dynamic strength performance in $1 \mathrm{R} M_{\mathrm{LE}}$ $(14 \%)$, whereas no significant changes were observed in $1 \mathrm{RM}_{\mathrm{BP}}(t=-1.249 ; P=0.240)$ (Table 1). After the training period, significant $(t=-2.259 ; P=0.047)$ increases were observed in power capacity performed by CMJ performance $(22.7 \%)$ (Table 1). In both functional tasks, the post-menopausal women showed significant improvements in performance after the training period in TUG $(-15.9 \%$ : 0.7 seconds; $t=4.858 ; P=0.001)$ and STS test $(26.5 \%$; $t=-2.971 ; P=0.014)($ Table 1$)$.

At the beginning of the program training, all participants showed normal ranges of NTX and ALP levels (Table 2). After the 16-weeks of combined exercise, results showed that there was no significant change in serum NTX (initial value [12.5 (0.52) $\mathrm{nmol} \mathrm{BCE} / \mathrm{mmol} \mathrm{Cr}$ to final value $11.5(0.40)$ nmol BCE $/ \mathrm{mmol} \mathrm{Cr}, 8 \% ; P=0.996]$ ) (Table 2), although, there was a significant increase in the serum ALP $(\mathrm{U} / \mathrm{l})$ levels $([21.8$ (2.21) $\mathrm{U} / \mathrm{L}$ to 24.7 (3.12) $\mathrm{U} / \mathrm{L}, 13.3 \%, t=-2.251 ; P=0.041]$ ) after the training program (Table 2 ).

\section{Discussion}

This study demonstrates that combined training focus on high-speed power training and aerobic exercise using a step platform in postmenopausal women induced significant improvements in maximal strength in $1 \mathrm{RM}_{\mathrm{LEG}}$ and $1 \mathrm{RM}_{\mathrm{BP}}$, CMJ power test and TUG and STS functional tests

Table 2 Markers of bone metabolism in postmenopausal women before and after 16 weeks of combined training.

\begin{tabular}{|c|c|c|c|}
\hline & $\mathrm{T} 1$ & $\mathrm{~T} 2$ & $P$ \\
\hline Measurement & $\mathrm{x} \pm \sigma$ & $\mathrm{x} \pm \sigma$ & $(\mathrm{T} 1-\mathrm{T} 2)$ \\
\hline B-ALP (U/L) & $21.8 \pm 2.21$ & $24.7 \pm 3.12$ & 0.041 \\
\hline $\begin{array}{l}\text { NTx (nmol } \\
\quad \text { BCE } / \mathrm{mmol} \mathrm{Cr})\end{array}$ & $12.5 \pm 0.52$ & $11.5 \pm 0.40$ & 0.996 \\
\hline
\end{tabular}


performance. There was no change in serum NTX level, but a clinically significant increase in SALP after 16 weeks.

After the training program, postmenopausal women showed clinically significant improvements in dynamic muscular strength in lower and upper limbs. These results are similar to those previously reported for older participants submitted to high-speed resistance programs [3]. Functional capacity also increases during the 16 weeks, supporting the fact that specific combined training may be an optimal resistance training intrasession to increase functional performance in postmenopausal. Considering that muscle power is more closely associated to functional task performance and risk of falling in postmenopausal women than maximal strength [19], these observations may help to explain the higher increase observed in TUG test and in STS performance. Usually, arising from a chair can be related to aging-associated neuromuscular wasting and morphological changes in the lower limbs [3]. Therefore, our results may reinforce the notion that combined training can be useful to counteract the muscular aging-associated wasting processes, and increase functional performance in every tasks such as climbing, sitting and rising from a chair [20]. This would help postmenopausal women to be more independent and maintain or increase their quality of life [2]. Also, the regulation of bone metabolism appears to be important to prevent the deterioration of bone mechanical properties [21]. The present study demonstrates that even though serum NTX levels were not decreased by combined exercise, there were significant increases in the serum SALP levels, showing the potential for combined exercise to positively affect bone quality in postmenopausal women. Previous studies have shown the positive effect of exercise on bone health in post-menopausal women, usually by a reduction in bone loss [22]. The participants of this study did not always show extremely high bone turnover. In our participants, the serum NTX level decreased and the ALP increased. Thus, the decrease in the serum NTX level might play an important role for the maintenance or increase of bone mass with the intervention with combined exercise as a significant antiresorptive effect of combined exercise on bone mass in postmenopausal women.

Our innovative design of program training, as the compliance of aerobic exercise performed by step platform, and training methodology include suggestions about exercise intensity that it should be above the aerobic threshold, corresponding to $60 \%$ to $70 \%$ of maximal oxygen uptake [23]. Thus, the complement of exercises under an exercise that stimulates lower limbs, can also promote significant improvements in muscle strength and body balance, a significant way to prevent falls in participants with gait disturbance as it is usually related to injuries in this population. This is an important outcome in line with meta-analysis in the elderly showing that exercise was effective in lowering the risk of falls and fall-related injuries in selected people, reducing health care costs [24].

Non-loading muscle exercise are ineffective for bone loss prevention [25], thus, it may be accepted that highintensity aerobics and resistance exercise seems to be more effective to increase bone metabolism than low- to moderate-intensity as walking exercise [26]. Nevertheless, the bone remodeling response to estrogen deficiency is an increase in the rate of bone remodeling activity and in the rate of bone resorption relative to formation, resulting in a net loss of bone mass [27]. The stimulus of physical activity is thought to first decrease the rate of turnover and secondly to increase bone formation. Endurance exercise training appears to be an insufficient stimulus to accomplish both tasks [28], which may explain why the present results after the combined training, induces the reduction in resorption/elimination rate.

Bone mineral density is controlled by bone turnover which is the balance between synthesis and resorption. Central to this balance is the ratio of osteoprotogerin (OPG) and Receptor activator of nuclear kappa-B ligand (RANKL). RANKL is important to osteoclastogenesis and maintains the osteoclast in an activated, bone resorbing mode [27]. OPG is the decoy receptor for RANKL, and so inhibits its bone resorptive activity [29]. Factors that are known to affect bone metabolism impact on the production of these molecules $[27,29,30]$ and so measurement of these molecules would be useful in future exercise studies.

Some limitations have been identified in this study namely, the participants were not randomly divided into the exercise and nonexercise (control) groups because it was impossible to complete the bone markers measurements in women's that do not participate in the exercise program and no measures were made regarding bone minerals in order to evaluate their proportion in local bone mass. However, this last item is not considered critical as the main purpose of this study was to examine the effect of exercise on bone formation and resorption markers, especially on the serum NTX level and ALP. Therefore, we believe that the duration of this study might be sufficient, if we focus on the effect of exercise on bone resorption markers such as serum NTX.

In conclusion, this study clearly demonstrates that the mechanism for the positive response of bone markers could be achieved by combined training exercise in postmenopausal women. Further, it appears to decrease the rate of remove/formation of bone turnover. Studies are needed to elucidate the efficacy of long term combined exercise response on the bone quality and the risk of falls as an exercise-related effect.

\section{Practical applications}

Postmenopausal women are an increasing segment of the population and consequently, interventions aiming to improve their quality of life are becoming critically important. Our results highlight that an innovative combined training intervention is an effective, safe, and efficient strategy to achieve significant and clinically relevant improvements in muscular and functional tasks performance. This effect is relevant to daily life activities of postmenopausal women, together with significant improvements in their bone metabolism.

\section{Disclosure of interest}

The authors declare that they have no competing interest. 


\section{References}

[1] Prince RL, Draper C. Bone and calcium. In: Lobo RA, Kelsey J, Marcus R, editors. Menopause. USA: Academic Press; 2001. p. 287-94.

[2] Bergstrom I, Parini P, Gustafsson SA, Andersson G, Brinck J. Physical training increases osteoprotegerin in postmenopausal women. J Bone Miner Metab 2012;30:202-7.

[3] Pereira A, Costa AM, Izquierdo M, Silva AJ, Marques MC, Williams JHH. J Sports Med Phys Fitness 2013;5:38-43.

[4] Pacifici R. Estrogen, cytokines, and pathogenesis of postmenopausal osteoporosis. J Bone Miner Res 1996;111: 1043-51.

[5] Moreira LD, Fronza FC, Dos Santos RN, Zach PL, Kunii IS, Hayashi $L F$, et al. The benefits of a high-intensity aquatic exercise program (HydrOS) for bone metabolism and bone mass of postmenopausal women. J Bone Miner Metab 2014;32:411-9.

[6] Mosti MP, Kaehler N, Stunes AK, Hoff J, Syversen U. Maximal strength training in postmenopausal women with osteoporosis or osteopenia. J Strength Cond Res 2013;27:2879-86.

[7] Silverman NE, Nicklas BJ, Ryan AS. Addition of aerobic exercise to a weight loss program increases BMD, with an associated reduction in inflammation in overweight postmenopausal women. Calc Tissue Int 2009;84:257-65.

[8] Klentrou P, Slack J, Roy B, Ladouceur M. Effects of exercise training with weighted vests on bone turnover and isokinetic strength in postmenopausal women. J Aging Phys Act 2007;15:287-99.

[9] Swezey RL, Swezey A, Adams J. Isometric progressive resistive exercise for osteoporosis. J Rheumatol 2000;27:1260-4.

[10] Parfitt AM, Travers R, Rauch F, Glorieux FH. Structural and cellular changes during bone growth in healthy children. Bone 2000;27:487-94.

[11] Garnero P. Bone markers in osteoporosis. Curr Osteop Rep 2009;7:84-90.

[12] Avbersek-Luznik I, Gmeiner Stopar T, Marc J. Activity or mass concentration of bone-specific alkaline phosphatase as a marker of bone formation. Clin Chem Lab Med 2007;45: 1014-8.

[13] Snow-Harter C, Bouxsein M, Lewis B, Charette S, Weinstein P, Marcus MD. Muscle strength has been suggested to increase the integrity and the bone's ability to withstand the biomechanical forces involved in muscular tasks. J Bone Miner Res 1990;5:589-95.

[14] Hazell T, Kenno K, Jakobi J. Functional benefit of power training for older adults. J Aging Phys Act 2007;15:349-59.

[15] Pereira A, Izquierdo M, Silva AJ, Costa AM, Gonzalez-Badillo $\mathrm{JJ}$, Marques MC. Muscle performance and functional capacity retention after a high-speed power training cessation. Exp Gerontol 2012;47:620-4.

[16] Rikli RE, Jones CJ. Development and validation of a functional fitness test for community-residing older adults. J Aging Phys Act 1999;7:129-61.
[17] Hallage T, Krause MP, Haile L, Miculis CP, Nagle EF, Reis RS, et al. The effects of 12 weeks of step aerobics training on functional fitness of elderly women. J Strength Cond Res 2010;24:2261-6.

[18] Karvonen MJ, Kentala E, Mustala O. The effects of training on heart rate: a longitudinal study. Ann Med Exp Biol Fenn 1957; 35:307-15.

[19] Skelton DA, Kennedy J, Rutherford OM. Explosive power and asymmetry in leg muscle function in frequent fallers and nonfallers aged over 65. Age Ageing 2002;31:119-25.

[20] Ensrud KE, Nevitt MC, Yunis C, Cauley JA, Seeley DG, Fox KM, et al. Correlates of impaired function in older women. J Am Geriatr Soc 1994;42:481-9.

[21] Yang C, Chen J, Wu F, Li J, Liang P, Zhang H, et al. Effects of 60day head-down bed rest on osteocalcin, glycolipid metabolism and their association with or without resistance training. Clin Endocrinol (Oxf) 2014;81:671-8.

[22] Oh EG, Yoo JY, Lee JE, Hyun SS, Ko IS, Chu SH. Effects of a 3month therapeutic lifestyle modification program to improve bone health in postmenopausal Korean women in a rural community: a randomized controlled trial. Res Nurs Health 2014;37:292-301.

[23] Chien MY, Wu YT, Hsu AT, Yang RS, Lai JS. Efficacy of a 24week aerobic exercise program for osteopenic postmenopausal women. Calc Tissue Int 2000;67:443-8.

[24] Chang T, Morton S, Rubenstein L, Mojica W, Maglione M, Suttorp $M$, et al. Interventions for the prevention of falls in older adults: systematic review and meta-analysis of randomised clinical trials. BMJ 2014:328, http://dx.doi.org/10.1136/ bmj.328.7441.680.

[25] Sinaki M, Wahner H, Offord K, Hodgson S. Efficacy on non loading exercises in prevention of vertebral bone loss in postmenopausal women:a controlled trial. Mayo Clinic Proc 1989;64:762-9.

[26] Behringer M, Gruetzner S, McCourt M, Mester J. Effects of weight-bearing activities on bone mineral content and density in children and adolescents: a meta-analysis. J Bone Miner Res 2014;29:467-78.

[27] O'Brien EA, Williams JHH, Marshall MJ. Osteoprotegerin is produced when prostaglandin synthesis is inhibited causing osteoclasts to detach from the surface of mouse parietal bone and attach to the endocranial membrane. Bone 2001;28:208-14.

[28] Murtezani A, Nevzati A, Ibraimi Z, Sllamniku S, Meka VS, Abazi $N$. The effect of land versus aquatic exercise program on bone mineral density and physical function in postmenopausal women with osteoporosis: a randomized controlled trial. Int J Orthop Rehabil 2014;16:319-25.

[29] O’Brien EA, Williams JHH, Marshall MJ. Osteoprotegerin ligand regulates osteoclast adherence to the bone surface in mouse calvaria. Biochem Biophys Res 2000;274:281-90.

[30] Humphrey EL, Williams JHH, Davie MWJ, Marshall MJ. Effects of dissociated glucocorticoids on OPG and RANKL in osteoblastic cells. Bone 2006;38:652-61. 